\title{
The method of Constructing a virtual scene with 3D Models in CarMaker
}

\author{
Jiansuo Yang ${ }^{1, a}$, Yuansheng Liu $^{2, b}$, Wenjuan Zhang ${ }^{1, c}$ \\ ${ }^{1}$ Computer Science and Technology, Beijing Union University, Beijing, 100101, China \\ ${ }^{2}$ Institute of Information, Beijing Union University, Beijing, 100101, China \\ aemail: geremyyang@163.com, bemail: xxtyuansheng@buu.edu.cn, cemail: \\ wenjuan_zhang007@sina.com
}

Keywords: 3DS MAX, CarMaker, 3D Model, Virtual Scene

\begin{abstract}
What the most important in a virtual test environment is the realism of a scene. This paper focuses on how to use some scene models set up by 3DS MAX in CarMaker which is simulation software for vehicle. Because CarMaker itself only can build some certain road models and some simple scene parameters, so it is the best way to construct a virtual scene combined with 3d models in CarMaker in order to increase the virtual scene's degrees of reality and reliability. CarMaker only can distinguish MOBJ and OBJ these two kinds of formats. But coordinate, color and material of the OBJ model derived from 3DS MAX will change in CarMaker after importing a OBJ model. It could be solved by changing some parameters in its .mtl file. Some methods and skills of 3DS MAX scene modelling are also proposed in this paper.
\end{abstract}

\section{Introduction of Modeling Tool}

3D Studio Max, referred to as the 3DS Max or MAX, is a 3D animation and rendering software developed by Discreet company (merged with Autodesk Co. Ltd.) based on PC system . 3DS MAX is first used in the computer game animation, then it is used to participate in the film's special effects production, such as the X-Men II, the last samurai.

CarMaker software a standard testing tool in virtual driving is the best one in the field of vehicle dynamics modeling, real-time simulation and hardware in the loop [1]. CarMaker which is the most ideal tool kit for vehicle testing engineering can be used to simulate and test car, truck and motorcycle's handling stability, braking performance, comfort, economy and power. CarMaker is a software used to assist people test the entire vehicle, vehicle auxiliary system, power transmission system(suspension, brakes etc.) and the whole vehicle controller(ECU) control strategy. CarMaker is completely running on PC [2][3]. And CarMaker can also be run in the MATLAB/Simulink environment which is very important for engineers to verify their algorithms.

Beiyuan campus test field of Beijing Union University are built in CarMaker with 3D models derived from 3DS MAX.

\section{Modeling Process}

In order to get a realistic simulation environment of Beiyuan test field, there are four steps to set up a scene model with 3DS MAX [4][5].

1. Subdivide scene modules. There are runways, traffic lights, conical barrels, several buildings on both sides and something else in Beiyuan test field. First of all, items in Beiyuan test field should be stripped out . Then determine each item's minimum module according to its front ,left, top views. Finally, it is to determine minimum module's size(CarMaker default standard unit is meter) of each item, color and material.

2. Make models for each item's minimum module.

3. Merge all small modules.

4. Export a file with OBJ format.

The whole process of modeling a scene CarMaker software combined with 3DS MAX is shown in Figure 1. In order to specify the process of the whole scene modeling, Beiyuan test field's traffic 
light is taken as an example to show how to model a item with 3DS MAX [6][7].

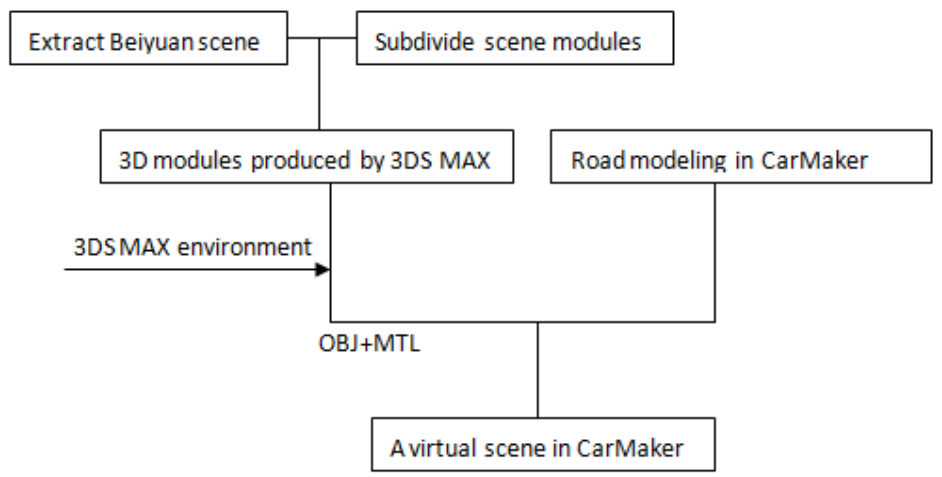

Fig.1 Modeling flow diagram

First, it is to subdivide scene modules. That establishing a virtual traffic light according to a real traffic light of Beiyuan test field which is shown in Figure 2 (a) is to illustrate the whole process of modeling a item. The first step is to segment modules, and traffic lights can be divided into specific lamp bracket, lamp box, lamp, signs and the top shield part of light frame. The second step is to determine the minimum size of each module: vertical lamp bracket's radius is 0.1 meters with height of 5.89 meters; the base of lamp stand is 0.321 meters long, 2.39 meters wide and 0.3 meters high; inclined support's radius is 0.08 meters with height of 3 meters; the top shield part of light frame is 1.5 meters long, 1.5 meters wide and 0.05 meters high; the middle bar half's diameter is 0.08 meters with height of 6.3 meters; the middle rail of the vertical supporting rod radius is 0.06 meters with height of 1 meter; the lamp box is 1.2 meters long, 0.5 meters wide and 0.5 meters high; light radius is 0.3 meters.

Open 3DS MAX and click the "custom" in the "unit settings" in which the choice of the metric system of units " $\mathrm{m}$ " and system settings inside the system unit scale are both set to "m". Draw a cylinder in the top view after choose "Cylinder" of "Geometry" in "Create Panel", and click "modify panel" to set cylinder's radius 0.1 meters with height of 5.89 meters and 1 segment of both height and end face, 32 edges. Draw another cylinder which is 0.08 meters long, 3 meters high and 32 edges and two cuboids whose size are $0.321 * 2.39 * 0.3$ and $1.5 * 1.5 * 0.005$ in the same way. Then get them together like Figure 2 (b) and (c).

Click on "Material editor"-“Standard”-“building”, then choose lamp bracket gray white color and the top shield part of light frame blue color in "Diffuse color" of "Metal - Brushed". All of these cylinders and cuboids should be grouped by clicking on "Group"-"Bunching" and named lamp bracket 01 . Finally, this $3 d$ model will be done after saving it as a file with MAX format.

The lateral support of traffic lights can be modeled in the same way. Now introduce how to make a lamp frame and the lamp. Select "new", draw a chamfer box in the top viewport and click on "Edit panel" to set its length 1.2 meters, width 0.5 meters and height 1 meter with length 4 length segments, 1 segment width and 8 height segments. Click on "Modify" - "Mesh editing" - "Edit polygon" and adjust the chamfer box's front side of 3 equal square in the "Edit polygon" - "edge". Click on "Polygon", choose a positive square and click on "Squeeze" to set its parameter as -0.3 and set parameter of "Profile" as -0.3. The lamp housing will be done after clicking on "modify" "subdivision" - "smoothing". Create a ball whose radius is 0.5 meters in the top viewport and select "Scaling" to scale this ball for a long ellipsoidal shape whose size is as same as the lamp housing. A simple lamp shown in Figure 2 (d) and (e) will be done well whiling moving the ball into the lamp housing.

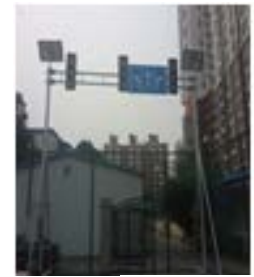

(a) Real traffic lights

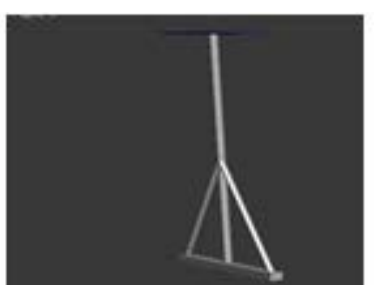

(b) Lamp bracket 1

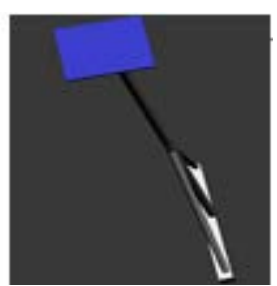

(c) Lamp bracket 2 


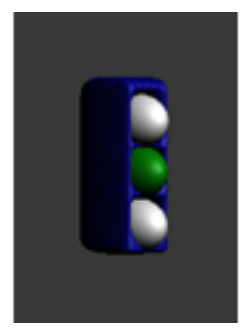

(d) The lamp's structure 1

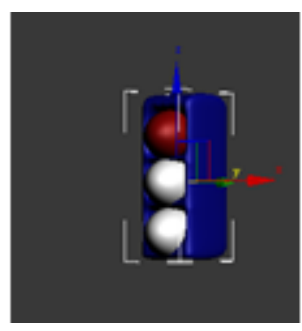

(e) The lamp’s structure 1

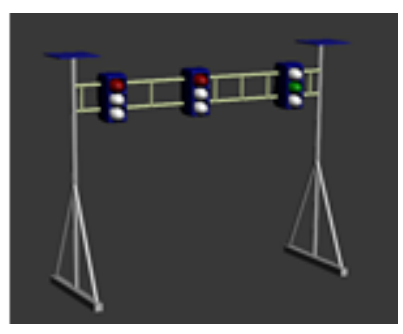

(f) A virtual traffic light

Fig.2 A traffic light of Beiyuan test field

What next to do is merging all items by clicking on "import" -"Merge”. Then the entire virutal traffic lights showed in Figure 2 (f) can be gained through roughly adjusting the position of each item.

\section{key Technology of Using 3D Models in CarMaker}

The first step to used 3d models in CarMaker is exporting these 3d models with OBJ format. Before exporting these models, determine all objects' coordinate because 3DS MAX's world coordinate is consistent with CarMaker's coordinate. And the starting point of plane map in CarMaker is the 1/2 Height of object instead of the initial point (0.0.0) in 3DS MAX. For instance, before exporting traffic lights, make traffic lights be a group and right click "Move" command to set its XYZ as $(0,0,4.45)$. Then click on "Export"-“Export the selected objects" and save them as files with OBJ format. Finally click on "OK" and choose "Flip YZ axis" in the pop-up "OBJ export options". If you don't flip their YZ axis, they will be a sleep state in CarMaker. There are two files that are .obj file and .mtl file. The .mtl file is very important because it includes some information such as color and material. And if you want to show the right color of model in CarMaker, you need to make sure that Tr parameter of .mtl file changes from " 0 " to " 1 ".

The second step is importing these $3 \mathrm{~d}$ models in CarMaker. First of all, find the installation file named IPG, then copy all .obj files and .mtl files to subdirectory "hil" - "win32-4.5.1" - "Movie" of folder IPG. Secondly, open CarMaker software and click on "Parameters" - "Traffic", then click on button "NEW" to add a 3d model which can be put anywhere through setting "StartPosition S-Y". If the $3 \mathrm{~d}$ model is a car, set its cruising speed by clicking on button "Start Velocity".

\section{Achievement Exhibition}

Click on "File" - "IPGMovie" in IPG home page, then the map of the traffic lights can be seen in the pop-up animation window which is shown in Figure 3.

Repeat the above operations and add other 3d models like playground, lookout, ball park in the virtual map in CarMaker. The comprehensive effect chart of Beiyuan test field is shown in Figure 4.

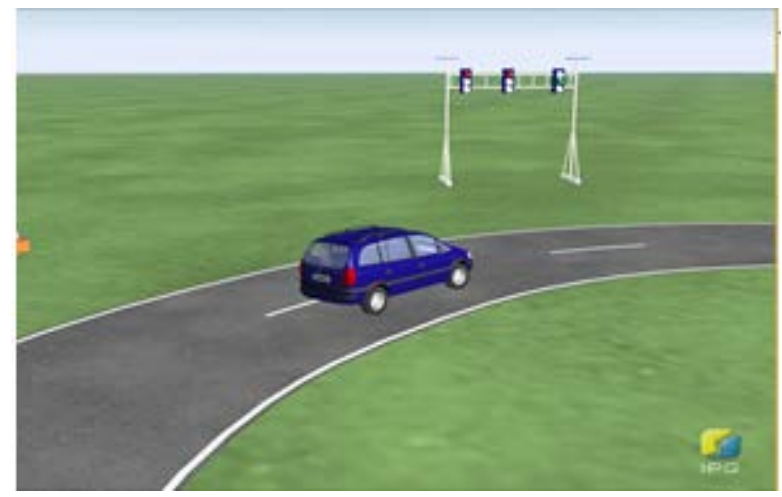

Fig.3 Animation window with traffic lights model 


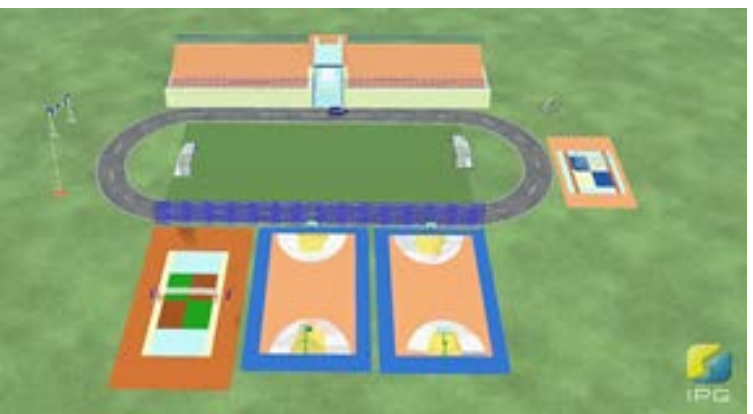

Fig.4 Comprehensive effect chart of Beiyuan test filed in CarMaker

\section{Conclusion}

The simulation system of unmanned technology requires a realistic scene. The paper realizes a virtual scene in CarMaker combined with 3DS MAX by taking Beiyuan test field of Beijing Union University as an example [8][9]. This kind of technology to build a virtual scene like the scene above will play a very important role in vehicle simulation system.

\section{Acknowledgement}

This work was financially supported by“The Project of Construction of Innovative Teams and Teacher Development for Universities and Colleges Under Beijing Municipality "and “The Importation and Development of High-Caliber Talents Project of Beijing Municipal Institutions "(CIT\&TCD201304074/IDHT20130513). And this paper's corresp- onding author is Yuansheng LIU whose email is xxtyuansheng@buu.edu.cn. His telephone number is +136 1119 2004 and his address is No.97 Beisihuan East Road,Chao Yang District, Beijing, P.R.China, 100101.

\section{References}

[1] Yanfang Huang. Virtual city modeling methods of 3DS MAX and MultiGen Creator combination[J]. Journal of Geomatics,2005,30(5)

[2] Liuxiang Dong. Volumetric 3D display technique based on image information derived from 3DS MAX[J]. Journal of Zhejiang University(Engineering Science),2005,30(11)

[3] Sundaravadivelu, K. Analysis of vehicle dynamics using co-simulation of AVL-CRUISE and CarMaker in ETAS RT environment[J]. 201402.

[4] Consoni, Flávia,From adaptation to complete vehicle design: A case study of product development capabilities in a CarMaker in Brazil.2006.

[5] Yanzhu Jiao. Research on 3DS MAX rendering and Photography Association Teaching[J]. Popular literature,2014,8

[6] Defa Hong, Wenxi Lu etl. Design and Realization of 3D virtual campus system[J]. Geospatial Information,2012,10(1)

[7] Ruixi Wang. The construction of virtual campus system based on Web. Shangdong: Shandong Agricultural University,2006

[8] Lili Wang, Weiling Wu. The creation of three-dimensional model based on 3ds max in virtual campus[J]. Forestry science and technology information,2009,41(1)

[9] Ning Liu, Weiming Zhen. The realization of 3D roaming system based on 3DS MAX[J]. Micro computer information,2011,8:225-226 